\title{
Limit behaviour of diffusion in high contrast periodic media and related Markov semigroups
}

\author{
A. Piatnitski ${ }^{1,2}$, S. Pirogov $^{2}$, E. Zhizhina ${ }^{2}$ \\ ${ }^{1}$ The Arctic university of Norway, campus Narvik, \\ P.O.Box 385, 8505 Narvik, Norway \\ 2 The Institute for Information Transmission Problems of RAS, \\ 19, Bolshoy Karetny per. 127051, Moscow \\ This paper is dedicated to the memory of Professor Vassily Zhikov. \\ Homogenization and high contrast media \\ were among his favorite subjects.
}

\begin{abstract}
The goal of the paper is to describe the large time behaviour of a symmetric diffusion in a highcontrast periodic environment and to characterize the limit process under the diffusive scaling. We consider separately the $C_{0}$ and $L^{2}$ settings.
\end{abstract}

\section{Introduction}

The paper deals with the large time behaviour of a diffusion and the corresponding semigroup defined in a high contrast periodic environment. Equivalently, we study the limit behaviour of a diffusion and the corresponding semigroup defined in a high contrast environment with a periodic microstructure on finite time intervals.

Elliptic and parabolic operators with high contrast rapidly oscillating periodic coefficients have been widely studied in homogenization theory. The first rigorous results for parabolic operators of this type were obtained in [6] and [2]. In particular, it was shown that, under proper choice of the scaling coefficient, the homogenized problem contains a non-local in time operator which reflects the so-called memory effect. Later on in [1], with the help of the two-scale convergence technique, the limit problem was written as a coupled system of parabolic PDEs in the space with a higher number of variables. In the works [9], [10] high contrast problems in domains with singular or asymptotically singular periodic geometry were considered. At present, there are many works in the existing mathematical literature that describe the effective behaviour of high contrast periodic media. Under proper scaling, in parabolic problems this usually results in the memory effect while homogenization of spectral problems leads to a non-linear dependence on the spectral parameter.

In this paper we deal with second order divergence form operators in $\mathbb{R}^{d}$. Each such an operator is a generator of a Markov semigroup. The corresponding Markov process (generalized diffusion) has continuous trajectories. However, the presence of a non-local term in the effective operator means that the limit dynamics of the coordinate process is not Markov.

The goal of this work is to equip the coordinate process with additional components in such a way that the dynamics of the enlarged process remains Markovian in the limit. We show that it is sufficient to combine the coordinate process with the position of the diffusion on the rescaled period. 
In the paper we consider the mentioned problem both in the spaces of continuous functions and in the $L^{2}$ framework. In both cases we introduce proper functional spaces, construct the limit semigroup, and prove the semigroup convergence.

We then use these results in order to obtain the convergence in law, in the path space, of the corresponding Markov process. It is interesting to observe that, although in the original processes the additional component is a function of the coordinate process, in the limit process this component is getting independent while the coordinate process becomes coupled with it.

In the $L^{2}$ setting, in addition to proving the semigroup convergence, we also describe the spectrum of the generator of the limit semigroup. Then the semigroup convergence in $L^{2}$ spaces allows us to provide some information about the limit behaviour of the spectrum of the original operators. In particular, we partly reprove the results on the convergence of the spectra obtained by V.Zhikov in [9] and [11] by means of two-scale convergence technique.

To our best knowledge, the questions considered in this paper have not been studied in the existing literature. In the discrete framework the results on scaling limits of symmetric random walks in a high contrast periodic environment were obtained in our previous work [7].

Our approach essentially relies on the approximation technique developed in [3] and the technique of periodic correctors [5].

\section{Problem setup}

Consider a symmetric diffusion operator in divergence form

$$
A_{\varepsilon} f(x)=\operatorname{div}\left(a_{\varepsilon}(x) \nabla f(x)\right), \quad a_{\varepsilon}(x)=\left\{a_{\varepsilon}^{i j}(x)\right\}_{i, j=1}^{d},
$$

where $a_{\varepsilon}^{i j}(x)$ are periodic for all $i, j=1, \ldots, d$ with period $[0,1)^{d}$. In what follows we identify $[0,1)^{d}$ periodic functions with functions on the unit torus $\mathbb{T}^{d}$ with a flat metric. We assume that

$$
a_{\varepsilon}(x)=\left\{\begin{array}{l}
a_{1}\left(\frac{x}{\varepsilon}\right), \quad x \in \mathbb{R}^{d} \backslash \varepsilon G^{\sharp}, \\
\varepsilon^{2} a_{2}\left(\frac{x}{\varepsilon}\right), \quad x \in \varepsilon G^{\sharp},
\end{array}\right.
$$

where $G \subset(0,1)^{d}$ is a smooth bounded simply connected domain such that $\bar{G} \subset(0,1)^{d}$, and $G^{\sharp}$ is a periodic extension of $G$ in $\mathbb{R}^{d}$. We assume furthermore that there exist $\alpha_{0}>0$ and $\alpha_{1} \geq \alpha_{0}$ such that

$$
\alpha_{0} \mathrm{I} \leq a_{1}^{i j}(y) \leq \alpha_{1} \mathrm{I} \text { if } y \in \mathbb{R}^{d} \backslash G^{\sharp}, \quad \alpha_{0} \mathrm{I} \leq a_{2}^{i j}(y) \leq \alpha_{1} \mathrm{I} \text { if } y \in G^{\sharp} .
$$

For the sake of brevity we denote $G_{\varepsilon}^{\sharp}=\varepsilon G^{\sharp}$ and $G^{c}=\mathbb{T}^{d} \backslash G$. Observe that under our assumptions both the diameter of a periodicity cell and the distance between neighbouring connected components of $G_{\varepsilon}^{\sharp}$ are of order $\varepsilon$.

Denote by $C_{0}\left(\mathbb{R}^{d}\right)$ the Banach space of continuous functions that vanish at infinity with the sup norm $\|u\|_{C}=\max _{x \in \mathbb{R}^{d}}|u(x)|$.

For each $\varepsilon>0$ we can interpret $A_{\varepsilon}$ as an unbounded operator in $C_{0}\left(\mathbb{R}^{d}\right)$ space or in $L^{2}\left(\mathbb{R}^{d}\right)$ space.

For the presentation simplicity we assume that both $a_{1}$ and $a_{2}$ are equal to the unit matrix. The case of generic symmetric periodic positive definite matrices $a_{1}(y)$ and $a_{2}(y)$ can be addressed in the same way as this special case.

It is well known (see, for instance, [4]) that for any $\varepsilon>0$ the operator $A_{\varepsilon}$ in $C_{0}\left(\mathbb{R}^{d}\right)$ is the generator of a strongly continuous positive contraction semigroup $T_{\varepsilon}(t)=e^{A_{\varepsilon} t}$. The domain of this operator is dense in $C_{0}\left(\mathbb{R}^{d}\right)$, the operator is closed, and for any $\lambda>0$ the resolvent $\left(\lambda-A_{\varepsilon}\right)^{-1}$ is a bounded operator. Moreover, $A_{\varepsilon}$ is the generator of a Markov process with continuous trajectories in $\mathbb{R}^{d}$, for this process we use the notation $Y_{\varepsilon}(t), t \geq 0$. 
In $L^{2}\left(\mathbb{R}^{d}\right)$ we introduce a domain of $A_{\varepsilon}$ by

$$
\begin{aligned}
D_{H}\left(A_{\varepsilon}\right)=\left\{f \in H^{1}\left(\mathbb{R}^{d}\right),\right. & f \in H^{2}\left(G_{\varepsilon}^{\sharp}\right) \cap H^{2}\left(\mathbb{R}^{d} \backslash G_{\varepsilon}^{\sharp}\right), \\
& \left.\left.\varepsilon^{2} \nabla f(x)\right|_{\partial G_{\varepsilon}^{\sharp}} \cdot n^{+}=-\left.\nabla f(x)\right|_{\partial G_{\varepsilon}^{\sharp}} \cdot n^{-}\right\}
\end{aligned}
$$

The last condition on $f \in D_{H}\left(A_{\varepsilon}\right)$ in (4) is the condition of continuity of the flow $a_{\varepsilon} \nabla f$ through the boundary $\partial G_{\varepsilon}^{\sharp}$.

Then $\left(A_{\varepsilon}, D_{H}\left(A_{\varepsilon}\right)\right)$ is a self-adjoint operator in $L^{2}\left(\mathbb{R}^{d}\right)$, and for any $\lambda>0$ the operator $\left(\lambda-A_{\varepsilon}\right)$ is coercive.

We consider separately the $C_{0}$ and $L^{2}$ settings.

\section{$2.1 \quad C_{0}$-setting}

In this section we consider $A_{\varepsilon}$ as an unbounded operator in $C_{0}\left(\mathbb{R}^{d}\right)$. For the set of continuous functions in $\mathbb{R}^{d}$ with a compact support we use the notation $C_{0}^{b}\left(\mathbb{R}^{d}\right)$. The domain $D\left(A_{\varepsilon}\right)$ of the operator $A_{\varepsilon}$ is the closure in the graph norm of the set of functions

$$
\begin{gathered}
\left\{f \in C_{0}^{b}\left(\mathbb{R}^{d}\right), f^{+}=\left.f\right|_{G_{\varepsilon}^{\sharp}} \in C^{\infty}\left(\overline{G_{\varepsilon}^{\sharp}}\right), f^{-}=\left.f\right|_{\mathbb{R}^{d} \backslash G_{\varepsilon}^{\sharp}} \in C^{\infty}\left(\overline{\mathbb{R}^{d} \backslash G_{\varepsilon}^{\sharp}}\right),\right. \\
\left.\varepsilon^{2} \nabla f^{+}(x)\right|_{\partial G_{\varepsilon}^{\sharp}} \cdot n^{+}=-\left.\nabla f^{-}(x)\right|_{\partial G_{\varepsilon}^{\sharp}} \cdot n^{-}, \\
\left.\left.\operatorname{div}\left(\varepsilon^{2} \nabla f^{+}(x)\right)\right|_{\partial G_{\varepsilon}^{\sharp}}=\left.\operatorname{div}\left(\nabla f^{-}(x)\right)\right|_{\partial G_{\varepsilon}^{\sharp}}\right\} .
\end{gathered}
$$

We describe now the limit Markov semigroup and its generator. Denote $E=\mathbb{R}^{d} \times G^{\star}$, where $G^{\star}=\{\star\} \cup G$, and $\{\star\}$ is a single point set which is identified with $G^{c}$. In $G^{\star}$ we introduce the topology generated by open sets in $G$ and $\delta$-neighborhoods of $\star$ defined by $O_{\delta}(\star)=O_{\delta}(\partial G), \delta>0$. Then $E$ is equipped with the product topology. A function $F \in C_{0}(E)$ can be written in a vector form

$$
F(x, \hat{y})= \begin{cases}f_{0}(x), & \text { if } x \in \mathbb{R}^{d}, \hat{y}=\star \\ \left.f_{1}(x, y)\right), & \text { if } x \in \mathbb{R}^{d}, \hat{y}=y \in G\end{cases}
$$

with $f_{0} \in C_{0}\left(\mathbb{R}^{d}\right), f_{1} \in C_{0}\left(\mathbb{R}^{d}, C(\bar{G})\right),\left.f_{1}(x, y)\right|_{y \in \partial G}=f_{0}(x)$. With this notation, for any $x \in \mathbb{R}^{d}$

$$
f_{0}(x)=F(x, \star), \quad f_{1}(x, y)=F(x, y) \text { for } y \in G .
$$

In what follows we identify a function $F(x, \hat{y})$ with the corresponding vector-function $\left(f_{0}(x), f_{1}(x, y)\right)$.

Let us consider in $C_{0}(E)$ an unbounded operator of the following form

$$
(A F)(x, \hat{y})=\left(\begin{array}{c}
\Theta \nabla \nabla f_{0}(x)+\frac{1}{\left|G^{c}\right|} \int_{\partial G} \frac{\partial f_{1}(x, y)}{\partial n_{y}^{-}} d \sigma(y) \\
\triangle_{y} f_{1}(x, y)
\end{array}\right),
$$

where a positive defined matrix $\Theta$ will be defined later on, $\sigma(y)$ is the element of the surface volume on $\partial G, n_{y}^{-}$is the (inner) normal to $\partial G$. Using the relation $n^{+}=-n^{-}$and the Stokes formula one can rewrite the operator (7) as follows:

$$
(A F)(x, \hat{y})=\left(\begin{array}{c}
\Theta \nabla \nabla f_{0}(x)-\frac{1}{\left|G^{c}\right|} \int_{G} \triangle_{y} f_{1}(x, y) d y \\
\triangle_{y} f_{1}(x, y)
\end{array}\right) .
$$


We define the domain $D(A)$ of the operator $A$ as a closure in the graph norm of the following set of functions:

$$
\begin{aligned}
& D_{A}=\left\{u_{0} \in C_{0}^{\infty}\left(\mathbb{R}^{d}\right), u_{1} \in C_{0}^{\infty}\left(\mathbb{R}^{d} ; C^{\infty}(\bar{G})\right),\left.u_{1}(x, y)\right|_{y \in \partial G}=u_{0}(x),\right. \\
&\left.\left.\triangle_{y} u_{1}(x, y)\right|_{y \in \partial G}=\Theta \nabla \nabla u_{0}(x)+\frac{1}{\left|G^{c}\right|} \int_{\partial G} \frac{\partial u_{1}(x, y)}{\partial n_{y}^{-}} d \sigma(y)\right\} .
\end{aligned}
$$

Lemma 2.1. The closure of the operator $A$ is a generator of a strongly continuous, positive, contraction semigroup $T(t)$ on $C_{0}(E)$.

Proof. In order to apply the Hille-Yosida theorem, and should make sure that:

1) $D(A)$ is dense in $C_{0}(E)$;

2) $A$ is a dissipative operator;

3) $\operatorname{Im}(\lambda-A)$ is dense in $C_{0}(E)$ for some $\lambda>0$.

The set of functions

$$
\mathcal{L}=\left\{u_{0} \in C_{0}^{\infty}\left(\mathbb{R}^{d}\right), u_{1} \in C_{0}^{\infty}\left(\mathbb{R}^{d}, C^{\infty}(\bar{G})\right),\left.u_{1}(x, y)\right|_{y \in \partial G}=u_{0}(x)\right\}
$$

is dense in $C_{0}(E)$. Then to prove the statement 1) it is sufficient to show that $D_{A}$ is dense in $\mathcal{L}$, i.e. for any $\delta>0$ and for any $u=\left(u_{0}, u_{1}\right) \in \mathcal{L}$ one can find $\hat{u}=\left(\hat{u}_{0}, \hat{u}_{1}\right) \in D_{A}$, such that $\|u-\hat{u}\|_{C(E)}<\delta$. We take $\hat{u}_{0}=u_{0}$ and construct $\hat{u}_{1}$ in such a way that the following relation holds:

$$
\left.\triangle_{y} \hat{u}_{1}(x, y)\right|_{y \in \partial G}-\frac{1}{\left|G^{c}\right|} \int_{\partial G} \frac{\partial \hat{u}_{1}(x, y)}{\partial n_{y}^{-}} d \sigma(y)=\Theta \nabla \nabla u_{0}(x) .
$$

To this end we set $\tilde{u}_{1}(x, y)=u_{1}(x, y) \psi(y)+u_{0}(x)(1-\psi(y))$ with

$$
\psi \in C_{0}^{\infty}(\bar{G}): \quad \psi(y) \equiv 1 \text { as } y \in G \backslash G_{\delta},
$$

where $G_{\delta}=\{y \in \bar{G}: \operatorname{dist}(x, \partial G)<\delta\}$. Then $\left.\tilde{u}_{1}(x, y)\right|_{\partial G}=u_{0}(x),\|u-\tilde{u}\|_{C(E)}<c_{1} \delta$, and the left hand side in (11) evaluated at $\tilde{u}$ is equal to 0 . Let $\chi(y)$ be a $C^{\infty}(\bar{G})$ function such that $\left.\chi(y)\right|_{y \in \partial G}=0$, $\left.\frac{\partial \chi(y)}{\partial n_{y}^{-}}\right|_{\partial G}=0,\left.\Delta \chi(y)\right|_{y \in \partial G}=1$, and $\|\chi\|_{C(\bar{G})}<\delta$. If we take $\hat{u}_{1}(x, y)=\tilde{u}_{1}(x, y)+\Theta \nabla \nabla u_{0}(x) \chi(y)$ then (11) holds and $\|u-\hat{u}\|<c_{2} \delta$, as required.

The fact that condition 2) holds is a direct consequence of the maximum principle:

$$
\|\lambda f-A f\|_{C_{0}(E)} \geq \lambda\|f\|_{C_{0}(E)}
$$

for any $f \in D(A)$ and any $\lambda>0$.

The third condition is a consequence of the following statement: there exists $\lambda>0$ such that for any $g=\left(g_{0}, g_{1}\right) \in C_{0}(E)$ the eqution $(\lambda-A) f=g$ has a solution $f \in D(A)$. To prove this statement we use (8) and rewrite the equation $(\lambda-A) f=g$ as follows

$$
\begin{gathered}
\lambda f_{0}-\Theta \nabla \nabla f_{0}(x)-\frac{1}{\left|G^{c}\right|} \int_{\partial G} \frac{\partial f_{1}(x, y)}{\partial n_{y}^{-}} d \sigma(y)=g_{0}(x), \\
\lambda f_{1}-\triangle_{y} f_{1}(x, y)=g_{1}(x, y) .
\end{gathered}
$$

Since the latter system is linear, $f_{1}(x, y)$ is a sum of the solutions of the following two problems:

$$
\left\{\begin{array}{l}
\lambda f_{1}^{I}-\triangle_{y} f_{1}^{I}(x, y)=0 \\
\left.f_{1}^{I}(x, y)\right|_{y \in \partial G}=f_{0}(x)
\end{array}\right.
$$


and

$$
\left\{\begin{array}{c}
\lambda f_{1}^{I I}-\triangle_{y} f_{1}^{I I}(x, y)=g_{1}(x, y) \\
\left.f_{1}^{I I}(x, y)\right|_{y \in \partial G}=0 .
\end{array}\right.
$$

Then the solution of the former problem takes the form $f_{1}^{I}(x, y)=f_{0}(x) \varphi(y)$, where $\varphi(y)$ satisfies the equation $\left(\lambda-\Delta_{y}\right) \varphi(y)=0$ in $G$, and the boundary condition $\left.\varphi(y)\right|_{y \in \partial G}=1$ on $\partial G$. The solution $f_{1}^{I I}(x, y)$ of the latter problem is a continuous function of $x$ and $y$ that vanishes as $x \rightarrow \infty$. The function

$$
f_{1}(x, y)=f_{0}(x) \varphi(y)+f_{1}^{I I}(x, y)
$$

satisfies the second equation in (12) and the boundary condition $\left.f_{1}(x, y)\right|_{y \in \partial G}=f_{0}(x)$. Inserting $f_{1}(x, y)$ into the first equation in (12) yields the following equation for $f_{0}$ :

$$
\lambda f_{0}-\Theta \nabla \nabla f_{0}(x)-\widehat{\lambda}_{0} f_{0}(x)=\tilde{g}_{0}(x) .
$$

with

$$
\widehat{\lambda}_{0}=\frac{1}{\left|G^{c}\right|} \int_{\partial G} \frac{\partial \varphi(y)}{\partial n_{y}^{-}} d \sigma(y), \quad \tilde{g}_{0}(x)=g_{0}(x)+\frac{1}{\left|G^{c}\right|} \int_{G} \Delta_{y} f_{1}^{I I}(x, y) d y
$$

Since $\Delta_{y} f_{1}^{I I}(x, y)=\lambda f_{1}^{I I}-g_{1}(x, y)$, then $\tilde{g}_{0} \in C_{0}\left(\mathbb{R}^{d}\right)$. Therefore, for any $\lambda>\widehat{\lambda}_{0}$ the latter equation has a solution $f_{0}(x) \in C_{0}\left(\mathbb{R}^{d}\right)$. Then $f_{1}$ defined in $(13)$ belongs to $C_{0}\left(\mathbb{R}^{d} ; C(\bar{G})\right)$, and we conclude that $\left(f_{0}, f_{1}\right) \in D(A)$. Therefore, the Hille-Yosida theorem applies, and the desired statement follows.

Corollary 1. For any $\lambda>\widehat{\lambda}_{0}$ the set $\left\{(\lambda-A) F: F \in D_{A}\right\}$ is dense in $C_{0}(E)$.

Proof. Consider equation (12) and choose a function $G=\left(g_{0}, g_{1}\right)$ on the right hand side such that $g_{0} \in C_{0}^{\infty}\left(\mathbb{R}^{d}\right), g_{1} \in C_{0}^{\infty}\left(\mathbb{R}^{d}, C^{\infty}(\bar{G})\right)$. By the same arguments as in the proof of Lemma 2.1, we conclude that the corresponding solution $F=\left(f_{0}, f_{1}\right)$ of problem (12) belongs to the Schwartz class in $x$, i.e. $f_{0} \in \mathcal{S}\left(\mathbb{R}^{d}\right), f_{1} \in \mathcal{S}\left(\mathbb{R}^{d}, C^{\infty}(\bar{G})\right)$.

For any $n \in \mathbb{Z}^{+}$denote by $\varphi_{n}$ a $C_{0}^{\infty}\left(\mathbb{R}^{d}\right)$ cut-off function such that

$$
0 \leq \varphi_{n} \leq 1, \quad \operatorname{supp} \varphi_{n} \subset\{x:|x| \leq n+1\}, \quad \varphi_{n}=1 \text { if }|x| \leq n, \quad\left|\nabla \varphi_{n}\right| \leq 2 .
$$

Then the function $\psi_{n}=2 \Theta \nabla f_{0} \nabla \varphi_{n}+f_{0} \Theta \nabla \nabla \varphi_{n}$ is an element of $C_{0}^{\infty}\left(\mathbb{R}^{d}\right)$, and, moreover, $\left\|\psi_{n}\right\|_{C\left(\mathbb{R}^{d}\right)} \rightarrow$ 0 , as $n \rightarrow \infty$. Let $\gamma(y)$ be a $C^{\infty}(\bar{G})$ function such that $\left.\gamma\right|_{\partial G}=0,\left.\nabla \gamma\right|_{\partial G}=0,\left.\Delta \gamma\right|_{\partial G}=1$. It is clear that

$$
F_{n}=\left(\varphi_{n}(x) f_{0}(x), \varphi_{n}(x) f_{1}(x, y)+\gamma(y) \psi_{n}(x)\right) \in D_{A}
$$

and that

$$
\varphi_{n}(x) f_{0}(x) \in C_{0}^{\infty}\left(\mathbb{R}^{d}\right), \quad \varphi_{n}(x) f_{1}(x, y)+\gamma(y) \psi_{n}(x) \in C_{0}^{\infty}\left(\mathbb{R}^{d}, C^{\infty}(\bar{G})\right) .
$$

Now straightforward computations show that $\left\|A F_{n}-G\right\|_{C_{0}(E)} \rightarrow 0$, as $n \rightarrow \infty$.

Remark 2.1. It follows from Corollary 1 that $D_{A}$ defined in (9) is a core of the operator $A$ in $C_{0}(E)$.

Define a bounded linear transformation $\pi_{\varepsilon}: C_{0}(E) \rightarrow C_{0}\left(\mathbb{R}^{d}\right)$ as follows:

$$
\left(\pi_{\varepsilon} F\right)(x)=\left\{\begin{array}{c}
f_{0}(x), \text { if } x \in \mathbb{R}^{d} \backslash G_{\varepsilon}^{\sharp} ; \\
f_{1}\left(x,\left\{\frac{x}{\varepsilon}\right\}\right), \text { if } x \in G_{\varepsilon}^{\sharp} .
\end{array}\right.
$$

here $\{\cdot\}$ stands for the fractional part. Then for all $\varepsilon \in(0,1)$

$$
\sup _{x \in \mathbb{R}^{d}}\left|\pi_{\varepsilon} F(x)\right|=\left\|\pi_{\varepsilon} F\right\|_{C_{0}\left(\mathbb{R}^{d}\right)} \leq \max _{k=0,1}\left\|f_{k}\right\|=\|F\|_{C_{0}(E)} .
$$


We are going to prove the semigroup convergence using the approximation theorem from [3] and correctors for the appropriate diffusions in periodic environment.

Theorem 2.1. For every $F \in C_{0}(E)$

$$
T_{\varepsilon}(t) \pi_{\varepsilon} F \rightarrow T(t) F \quad\left(\text { i.e. }\left\|T_{\varepsilon}(t) \pi_{\varepsilon} F-\pi_{\varepsilon} T(t) F\right\|_{C_{0}\left(\mathbb{R}^{d}\right)} \rightarrow 0\right) \quad \text { for all } \quad t \geq 0
$$

as $\varepsilon \rightarrow 0$.

The proof of (15) relies on the following approximation theorem [3, Theorem 6.1, Ch.1].

Theorem (see [3]). For $n=1,2, \ldots$, let $T_{n}(t)$ and $T(t)$ be strongly continuous contraction semigroups on Banach space $\mathcal{L}_{n}$ and $\mathcal{L}$, with generators $A_{n}$ and $A$. Let $D$ be a core for $A$. Then the following are equivalent:

a) For each $f \in \mathcal{L}, T_{n}(t) \pi_{n} f \rightarrow T(t) f$ for all $t \geq 0$.

b) For each $f \in D$, there exists $f_{n} \in \mathcal{L}_{n}$ for each $n \geq 1$ such that $f_{n} \rightarrow f$ and $A_{n} f_{n} \rightarrow A f$.

According to this theorem the semigroups convergence (15) is a consequence of the following statement:

Lemma 2.2. Let the generators $A$ and $A_{\varepsilon}$ of the strongly continuous, positive, contraction semigroups $T(t)$ and $T_{\varepsilon}(t)$ be defined by (7) and (1), (2), (5), respectively, and let a core $D_{A} \subset C_{0}(E)$ of the generator $A$ be defined by (9).

Then for any $F=\left(f_{0}, f_{1}\right) \in D_{A}$, there exists $F_{\varepsilon} \in D\left(A_{\varepsilon}\right)$ such that

$$
\left\|F_{\varepsilon}-\pi_{\varepsilon} F\right\|_{C_{0}\left(\mathbb{R}^{d}\right)} \rightarrow 0 \quad \text { and } \quad\left\|A_{\varepsilon} F_{\varepsilon}-\pi_{\varepsilon} A F\right\|_{C_{0}\left(\mathbb{R}^{d}\right)} \rightarrow 0, \quad \text { as } \varepsilon \rightarrow 0,
$$

where $\pi_{\varepsilon}: C_{0}(E) \rightarrow C_{0}\left(\mathbb{R}^{d}\right)$ is a bounded linear transformation defined by (14).

Proof. The proof relies on the correctors technique widely used in the homogenization theory. In order to make (16) hold we construct the following family of functions $F_{\varepsilon}$ :

$$
F_{\varepsilon}(x)=\left\{\begin{array}{l}
f_{0}(x)+\varepsilon\left(\nabla f_{0}(x), h\left(\frac{x}{\varepsilon}\right)\right)+\varepsilon^{2}\left(\nabla \nabla f_{0}(x), g\left(\frac{x}{\varepsilon}\right)\right)+\varepsilon^{2} \Phi\left(x, \frac{x}{\varepsilon}\right), x \in \mathbb{R}^{d} \backslash G_{\varepsilon}^{\sharp}, \\
f_{1}\left(x, \frac{x}{\varepsilon}\right)+\varepsilon \phi_{\varepsilon}\left(x, \frac{x}{\varepsilon}\right), x \in G_{\varepsilon}^{\sharp},
\end{array}\right.
$$

where $h(\xi), g(\xi), \Phi(x, \xi)$ and $\phi_{\varepsilon}(x, \xi)$ are periodic smooth functions of $\xi$ (so-called correctors) defined on the periodicity cell, $h(\xi)$ is the periodic vector function, $g(\xi)$ is the periodic matrix function. A corrector $\phi_{\varepsilon}(x, \xi)$ has been introduced in order to ensure the continuity of the function $F_{\varepsilon}$ on the boundary $\partial G_{\varepsilon}^{\sharp}$. In addition, the functions $\Phi(x, \xi), \phi_{\varepsilon}(x, \xi)$ are smooth in $x$ and tend to zero as $x \rightarrow \infty$. Moreover, $\left\|\phi_{\varepsilon}\right\|_{C_{0}^{2}\left(\mathbb{R}^{d} \times \bar{G}\right)} \leq C$ with a constant $C$ that does not depend on $\varepsilon$.

The structure (17) of the function $F_{\varepsilon}$ immediately implies the first convergence in (16):

$$
\sup _{x}\left|F_{\varepsilon}(x)-\pi_{\varepsilon} F(x)\right|=O(\varepsilon) \rightarrow 0 \quad \text { as } \varepsilon \rightarrow 0 .
$$

In order to justify the second convergence in (16) we deduce from (7) and (14) that, for each $\varepsilon>0$

$$
\left\|A_{\varepsilon} F_{\varepsilon}-\pi_{\varepsilon} A F\right\|_{C_{0}\left(\mathbb{R}^{d}\right)}=
$$




$$
\max \left\{\max _{x \in \mathbb{R}^{d} \backslash G_{\varepsilon}^{\sharp}}\left|A_{\varepsilon} F_{\varepsilon}(x)-\left(\Theta \cdot \nabla \nabla f_{0}(x)+\Upsilon(x)\right)\right|, \max _{x \in G_{\varepsilon}^{\sharp}, \xi \in G}\left|A_{\varepsilon} F_{\varepsilon}(x)-\triangle_{\xi} f_{1}(x, \xi)\right|\right\},
$$

where $\Upsilon(x)=\frac{1}{\left|G^{c}\right|} \int_{\partial G} \frac{\partial f_{1}(x, \xi)}{\partial n_{\xi}^{-}} d \sigma(\xi)$. Thus we have to prove that both terms on the right-hand side of (18) tend to 0 as $\varepsilon \rightarrow 0$.

Using (17) and the formula

$$
\frac{\partial}{\partial x} f\left(x, \frac{x}{\varepsilon}\right)=\left.\left(\frac{\partial}{\partial x} f(x, \xi)+\frac{1}{\varepsilon} \frac{\partial}{\partial \xi} f(x, \xi)\right)\right|_{\xi=\frac{x}{\varepsilon}},
$$

we get

$$
\begin{gathered}
\left(A_{\varepsilon} F_{\varepsilon}\right)(x)=\left(\triangle f_{0}(x)+2 \nabla \nabla f_{0}(x) \nabla_{\xi} h(\xi)\right. \\
\left.+\frac{1}{\varepsilon} \nabla f_{0}(x) \triangle_{\xi} h(\xi)+\nabla \nabla f_{0}(x) \triangle_{\xi} g(\xi)+\triangle_{\xi} \Phi(x, \xi)+\varepsilon \Xi_{\varepsilon}(x, \xi)\right)\left.\right|_{\xi=\frac{x}{\varepsilon}},
\end{gathered}
$$

if $x \in \mathbb{R}^{d} \backslash G_{\varepsilon}^{\sharp}$, and

$$
\left(A_{\varepsilon} F_{\varepsilon}\right)(x)=\varepsilon^{2} \triangle_{x}\left(f_{1}\left(x, \frac{x}{\varepsilon}\right)+\varepsilon \phi_{\varepsilon}\left(x, \frac{x}{\varepsilon}\right)\right)=\left.\left(\triangle_{\xi} f_{1}(x, \xi)+\varepsilon \Psi_{\varepsilon}(x, \xi)\right)\right|_{\xi=\frac{x}{\varepsilon}},
$$

if $x \in G_{\varepsilon}^{\sharp}$, where

$\Xi_{\varepsilon}(x, \xi)=\Delta \nabla f_{0}(x) \cdot h(\xi)+2 \nabla \nabla \nabla f_{0}(x) \nabla_{\xi} g(\xi)+\varepsilon \Delta \nabla \nabla f_{0}(x) g(\xi)+\varepsilon \Delta_{x} \Phi(x, \xi)+2 \nabla_{x} \cdot \nabla_{\xi} \Phi(x, \xi)$, and

$$
\Psi_{\varepsilon}(x, \xi)=\varepsilon \Delta_{x} f_{1}(x, \xi)+2 \nabla_{x} \cdot \nabla_{\xi} f_{1}(x, \xi)+\varepsilon^{2} \Delta_{x} \phi_{\varepsilon}(x, \xi)+2 \varepsilon \nabla_{x} \cdot \nabla_{\xi} \phi_{\varepsilon}(x, \xi)+\Delta_{\xi} \phi_{\varepsilon}(x, \xi) .
$$

Moreover, since $F_{\varepsilon}$ should belong to $D\left(A_{\varepsilon}\right)$ the following conditions should be fulfilled for all $x \in \partial G_{\varepsilon}^{\sharp}:$

1) the continuity of functions

$$
f_{0}(x)+\varepsilon\left(\nabla f_{0}(x), h(\xi)\right)+\varepsilon^{2}\left(\nabla \nabla f_{0}(x), g(\xi)\right)+\left.\varepsilon^{2} \Phi(x, \xi)\right|_{\xi=\frac{x}{\varepsilon}}=f_{1}(x, \xi)+\left.\varepsilon \phi_{\varepsilon}(x, \xi)\right|_{\xi=\frac{x}{\varepsilon}} ;
$$

2) the continuity of fluxes

$$
\begin{gathered}
\left.\nabla_{x}\left(f_{0}(x)+\varepsilon\left(\nabla f_{0}(x), h\left(\frac{x}{\varepsilon}\right)\right)+\varepsilon^{2}\left(\nabla \nabla f_{0}(x), g\left(\frac{x}{\varepsilon}\right)\right)+\varepsilon^{2} \Phi\left(x, \frac{x}{\varepsilon}\right)\right)\right|_{x \in \partial G_{\varepsilon}^{\sharp}} \cdot n^{-}= \\
-\left.\varepsilon^{2} \nabla_{x}\left(f_{1}\left(x, \frac{x}{\varepsilon}\right)+\varepsilon \phi_{\varepsilon}\left(x, \frac{x}{\varepsilon}\right)\right)\right|_{x \in \partial G_{\varepsilon}^{\sharp}} \cdot n^{+},
\end{gathered}
$$

3) the continuity of images

$$
\begin{gathered}
\operatorname{div}\left(\left.a_{1}\left(\frac{x}{\varepsilon}\right) \nabla\left(f_{0}(x)+\varepsilon\left(\nabla f_{0}(x), h\left(\frac{x}{\varepsilon}\right)\right)+\varepsilon^{2}\left(\nabla \nabla f_{0}(x), g\left(\frac{x}{\varepsilon}\right)\right)+\varepsilon^{2} \Phi\left(x, \frac{x}{\varepsilon}\right)\right)\right|_{x \in \partial G_{\varepsilon}^{\sharp}}=\right. \\
\varepsilon^{2} \operatorname{div}\left(\left.a_{2}\left(\frac{x}{\varepsilon}\right) \nabla\left(f_{1}\left(x, \frac{x}{\varepsilon}\right)+\varepsilon \phi_{\varepsilon}\left(x, \frac{x}{\varepsilon}\right)\right)\right|_{x \in \partial G_{\varepsilon}^{\sharp}}\right.
\end{gathered}
$$

where $n^{+}, n^{-}$are exterior and interior unit normal to $\partial G$ respectively (and $n^{+}=-n^{-}$). The relation in 2) can be rewritten as

$$
\begin{gathered}
\left(\nabla f_{0}(x)+\varepsilon \nabla \nabla f_{0}(x) h(\xi)+\nabla_{\xi}\left(\nabla f_{0}(x) h(\xi)\right)+\varepsilon^{2} \nabla \nabla \nabla f_{0}(x) g(\xi)\right. \\
\left.+\varepsilon \nabla_{\xi}\left(\nabla \nabla f_{0}(x) g(\xi)\right)+\varepsilon^{2} \nabla_{x} \Phi(x, \xi)+\varepsilon \nabla_{\xi} \Phi(x, \xi)\right)\left.\right|_{\xi \in \partial G} \cdot n^{-} \\
=-\left.\left(\varepsilon^{2} \nabla_{x} f_{1}(x, \xi)+\varepsilon \nabla_{\xi} f_{1}(x, \xi)+\varepsilon^{2} \nabla_{\xi} \phi_{\varepsilon}(x, \xi)+\varepsilon^{3} \nabla_{x} \phi_{\varepsilon}(x, \xi)\right)\right|_{\xi \in \partial G} \cdot n^{+} .
\end{gathered}
$$


To obtain equations on the periodic function $h(\xi)$ we collect the terms of the order $\varepsilon^{-1}$ in (19) and of the order $\varepsilon^{0}$ in (22). This yields

$$
\nabla f_{0}(x) \triangle_{\xi} h(\xi)=0, \xi \in G^{c}, \quad\left(\nabla f_{0}(x)+\nabla f_{0}(x) \nabla_{\xi} h(\xi)\right) \cdot n^{-}=0, \xi \in \partial G^{c} .
$$

Since $f_{0}$ does not depend on $\xi$, this problem can be rewritten as follows:

$$
\triangle h(\xi)=0, \quad \xi \in G^{c}, \quad \nabla_{\xi} h(\xi) \cdot n^{-}=-n^{-}, \quad \xi \in \partial G^{c},
$$

and, in the coordinate form,

$$
\triangle h^{j}(\xi)=0, \quad \xi \in G^{c}, \quad \nabla_{\xi} h^{j}(\xi) \cdot n^{-}=-n_{j}^{-}, \quad \xi \in \partial G^{c} .
$$

It is easy to check that the compatibility condition is valid:

$$
\int_{\partial G^{c}} n_{\xi}^{-} d \sigma(\xi)=0
$$

consequently, equation (24) has a unique up to an additive constant vector solution $h(\xi)$. Since $G$ is a smooth domain, the function $h(\xi)$ is smooth.

At the next step we collect the terms of order $\varepsilon^{0}$ on the right-hand side of (19) and equate them to $\Theta \nabla \nabla f_{0}(x)+\Upsilon(x)$ in order to make the first term in the figure brackets in (18) small:

$$
\triangle f_{0}(x)+2 \nabla \nabla f_{0}(x) \cdot \nabla_{\xi} h(\xi)+\nabla \nabla f_{0}(x) \triangle_{\xi} g(\xi)+\triangle_{\xi} \Phi(x, \xi)=\Theta \nabla \nabla f_{0}(x)+\Upsilon(x) .
$$

We also collect the terms of order $\varepsilon^{1}$ in (22):

$$
\left.\varepsilon\left(\nabla \nabla f_{0}(x) h(\xi)+\nabla_{\xi}\left(\nabla \nabla f_{0}(x) g(\xi)\right)+\nabla_{\xi} \Phi(x, \xi)\right)\right|_{\xi \in \partial G} \cdot n^{-}=-\left.\varepsilon \nabla_{\xi} f_{1}(x, \xi)\right|_{\xi \in \partial G} \cdot n^{+} .
$$

Notice that so far the constant matrix $\Theta$ and the function $\Upsilon$ have not been specified.

Collecting all the terms in (25)-(26) that contain the second order derivatives of $f_{0}$, we arrive at the following problem for the periodic matrix function $g(\xi)$ :

$$
\begin{gathered}
\triangle f_{0}(x)+2 \nabla \nabla f_{0}(x) \cdot \nabla_{\xi} h(\xi)+\nabla \nabla f_{0}(x) \triangle_{\xi} g(\xi)=\Theta \nabla \nabla f_{0}(x), \quad \xi \in G^{c}, \\
\left(\nabla \nabla f_{0}(x) h(\xi)+\nabla \nabla f_{0}(x) \nabla_{\xi} g(\xi)\right) \cdot n^{-}=0, \quad \xi \in \partial G^{c} .
\end{gathered}
$$

The solvability condition for this problem reads

$$
\int_{G^{c}}\left(-\left(E+2 \nabla_{\xi} h(\xi)\right)+\Theta\right) d \xi+\int_{\partial G^{c}} h(\xi) \otimes n^{-} d \sigma(\xi)=0 .
$$

This allows us to define the constant matrix $\Theta$ as follows:

$$
\Theta\left|G^{c}\right|=\int_{G^{c}}\left(E+2 \nabla_{\xi} h(\xi)\right) d \xi-\int_{\partial G^{c}} h(\xi) \otimes n^{-} d \sigma(\xi) .
$$

Under relation (27) the function $g(\xi)$ exists and is unique (up to an additional constant matrix).

Let us prove that the matrix $\Theta$ is positive definite. Using integration by parts formula, we obtain

$$
\int_{G^{c}} \nabla_{\xi} h(\xi) d \xi=\int_{\partial G^{c}} h(\xi) \otimes n^{-} d \sigma(\xi) .
$$


Thus, $\Theta\left|G^{c}\right|=\int_{G^{c}}\left(E+\nabla_{\xi} h(\xi)\right) d \xi$. Since $\left(E+\nabla_{\xi} h(\xi)\right)\left(E+\nabla_{\xi} h(\xi)\right)^{*}>0$, then it is sufficient to show that

$$
\int_{G^{c}}\left(E+\nabla_{\xi} h(\xi)\right)\left(\nabla_{\xi} h(\xi)\right)^{*} d \xi=0
$$

The last equality follows from integration by parts formula and relation (24) for the function $h$. Indeed,

$$
\int_{G^{c}}\left(E+\nabla_{\xi} h(\xi)\right)\left(\nabla_{\xi} h(\xi)\right)^{*} d \xi=-\int_{\partial G^{c}}\left(E+\nabla_{\xi} h(\xi)\right) n^{-} \otimes h(\xi) d \sigma(\xi)-\int_{G^{c}} \triangle h(\xi) \otimes h(\xi) d \xi=0 .
$$

Collecting the remaining terms in (25) and (26) we obtain the following problem for the function $\Phi(x, \xi)$ :

$$
\triangle_{\xi} \Phi(x, \xi)=\Upsilon(x), \quad \xi \in G^{c}, \quad \nabla_{\xi} \Phi(x, \xi) \cdot n^{-}=\nabla_{\xi} f_{1}(x, \xi) \cdot n^{-}, \quad \xi \in \partial G^{c} .
$$

The solvability condition for problem (28) gives the expression for $\Upsilon(x)$ :

$$
\Upsilon(x)\left|G^{c}\right|=\int_{\partial G} \nabla_{\xi} f_{1}(x, \xi) \cdot n_{\xi}^{-} d \sigma(\xi)=-\int_{\partial G} \nabla_{\xi} f_{1}(x, \xi) \cdot n_{\xi}^{+} d \sigma(\xi) .
$$

Thus, the function $\Upsilon(x)$ and the solution $\Phi(x, \xi)$ are uniquely defined. Moreover, $\Upsilon \in C_{0}^{\infty}\left(\mathbb{R}^{d}\right)$ and $\Phi(x, \xi) \in C_{0}^{\infty}\left(\mathbb{R}^{d} ; C^{\infty}\left(G^{c}\right)\right)$.

Our expansions have been designed in such a way, that

$$
\max _{x \in \mathbb{R}^{d} \backslash G_{\varepsilon}^{\sharp}}\left|A_{\varepsilon} F_{\varepsilon}(x)-\left(\Theta \cdot \nabla \nabla f_{0}(x)+\Upsilon(x)\right)\right| \leq C \varepsilon, \quad \max _{x \in G_{\varepsilon}^{\sharp}, \xi \in G}\left|A_{\varepsilon} F_{\varepsilon}(x)-\triangle_{\xi} f_{1}(x, \xi)\right| \leq C \varepsilon .
$$

However, the continuity conditions 1) - 3) need not be fulfilled.

The last step of the proof is to define a function $\phi_{\varepsilon}(x, \xi)$ in such a way that conditions 1) - 3) hold true. The main purpose of this function is to compensate the discrepancy between the inner and the outer expansions for the function $F_{\varepsilon}$ at the boundary $\partial G_{\varepsilon}^{\sharp}$. Condition 1 ) leads to the relation

$$
\phi_{\varepsilon}(x, \xi)=\nabla f_{0}(x) h(\xi)+\varepsilon \nabla \nabla f_{0}(x) g(\xi)+\varepsilon \Phi(x, \xi), \quad \xi \in \partial G .
$$

The continuity of fluxes condition (condition 2)) reads

$$
\left(\nabla \nabla \nabla f_{0}(x) g(\xi)+\nabla_{x} \Phi(x, \xi)\right) \cdot n^{-}+\left(\nabla_{x} f_{1}(x, \xi)+\varepsilon \nabla_{x} \phi_{\varepsilon}(x, \xi)\right) \cdot n^{+}=-\nabla_{\xi} \phi_{\varepsilon}(x, \xi) \cdot n^{+}, \quad \xi \in \partial G .
$$

Here $\nabla_{x} \phi_{\varepsilon}(x, \xi)$ can be found from relation (30).

Condition 3) takes the form

$$
\Xi_{\varepsilon}(x, \xi)=\Psi_{\varepsilon}(x, \xi), \quad \xi \in \partial G ;
$$

here we have used equations (23), (25), and the fact that $f=\left(f_{0}(x), f_{1}(x, \xi)\right) \in D_{A}$. Observe that in the relation (32) all the terms have already been specified except for the term $\Delta_{\xi} \phi_{\varepsilon}(x, \xi)$.

Since the boundary $\partial G$ is smooth, we can introduce the local coordinates in the vicinity of $\partial G$ so that one of these coordinates coincides with the normal and the others are coordinates on $\partial G$. In these coordinates one can extend the function $\phi_{\varepsilon}(x, \xi)$ to $\mathbb{R}^{d} \times \bar{G}$ in such a way that it satisfies conditions (30) - (32) and the additional condition

$$
\sup _{\xi \in G, x \in \mathbb{R}^{d}}\left\{\nabla_{\xi} \nabla_{\xi} \phi_{\varepsilon}, \nabla_{x} \nabla_{\xi} \phi_{\varepsilon}, \nabla_{x} \nabla_{x} \phi_{\varepsilon}\right\} \leq C_{0} .
$$

This construction is explicit and we leave the details to the reader.

This completes the proof of Lemma 2.2 . 
As was explained above, relation (15) is a consequence of Lemma 2.2.

According to the general results, see e.g. [4], there exist Markov processes $Y_{\varepsilon}(t)$ with continuous trajectories in $\mathbb{R}^{d}$ (a generalized diffusion process) generated by operators $A_{\varepsilon}$. In Theorem 2.1 we justified the convergence of the corresponding semigroups, and our next problem is to prove the existence of the limit process $X(t)$ in $E$ with sample paths in $D_{E}[0, \infty)$ and to prove the convergence of the processes in the Skorokhod topology of $D_{E}[0, \infty)$. However the process $Y_{\varepsilon}(t)$ lives in $\mathbb{R}^{d}$, and to formulate the statement about convergence of the processes we should first equip the coordinate process $Y_{\varepsilon}(t)$ with an additional variable $k_{\varepsilon}(y) \in G^{\star}$, that specifies the position of $Y_{\varepsilon}(t)$ in the period $\mathbb{T}^{d}$. We define a process $X_{\varepsilon}(t)$ with trajectories in $E=\mathbb{R}^{d} \times G^{\star}$ as follows

$$
X_{\varepsilon}(t)=\left(Y_{\varepsilon}(t), k_{\varepsilon}\left(Y_{\varepsilon}(t)\right)\right) \text {, where } k_{\varepsilon}(y)=\left\{\frac{y}{\varepsilon}\right\} \in G \text { if } y \in G_{\varepsilon}^{\sharp} \text { and } k_{\varepsilon}(y)=\star \text { if } y \in \mathbb{R}^{d} \backslash G_{\varepsilon}^{\sharp} \text {. }
$$

Remark 2.2. The semigroup $T(t)$ introduced in Lemma 2.1 is a Feller semigroup on $C_{0}(E)$.

Theorem 2.2. For any initial distribution $\nu \in \mathcal{P}(E)$ there exists a Markov process $X(t)$ corresponding to the semigroup $T(t): C_{0}(E) \rightarrow C_{0}(E)$ with generator $A$ defined by (7), (9) and with sample paths in $D_{E}[0, \infty)$.

If $\nu$ is the limit law of $X_{\varepsilon}(0)$, then $X_{\varepsilon}(t)$ converges in distribution to $X(t)$ as $\varepsilon \rightarrow 0$ in the Skorokhod topology of $D_{E}[0, \infty)$ :

$$
X_{\varepsilon}(t) \Rightarrow X(t) \quad \text { in } D_{E}[0, \infty) \text { as } \varepsilon \rightarrow 0 .
$$

Proof. To prove convergence (34) we combine the convergence of the finite dimensional distributions of $X_{\varepsilon}(t)$ (that is a consequence of Theorem 2.1) and the tightness of $X_{\varepsilon}(t)$ in $D_{E}[0, \infty)$.

We apply here Theorem 2.11 from [3], Chapter 4. For the reader convenience we formulate it here.

Theorem (see [3]). Let $M, M_{1}, M_{2}, \ldots$ be metric spaces with $M$ locally compact and separable. For $n=1,2, \ldots$ let $\eta_{n}: M_{n} \rightarrow M$ be measurable, let $T_{n}(t)$ be a semigroup on $B\left(M_{n}\right)$ (the Banach space of bounded functions with the sup-norm), and suppose $Y_{n}$ is a Markov process in $M_{n}$ corresponding to $T_{n}(t)$ such that $X_{n}=\eta_{n} \circ Y_{n}$ has sample paths in $D_{M}[0, \infty)$. Define $\pi_{n}: B(M) \rightarrow B\left(M_{n}\right)$ by $\pi_{n} f=f \circ \eta_{n}$. Suppose that $T(t)$ is a Feller semigroup on $C_{0}(M)$ and that for each $f \in C_{0}(M)$ and $t \geq 0$

$$
T_{n}(t) \pi_{n} f \rightarrow T(t) f, \quad \text { i.e. }\left\|T_{n}(t) \pi_{n} f-\pi_{n} T(t) f\right\| \rightarrow 0 .
$$

If $\left\{X_{n}(0)\right\}$ has limiting distribution $\nu \in \mathcal{P}(M)$, then there is a Markov process $X$ corresponding to $T(t)$ with initial distribution $\nu$ and sample paths in $D_{M}[0, \infty)$, and $X_{n} \Rightarrow X$.

We now adapt the constructions of this theorem to our framework. Let $n=\frac{1}{\varepsilon}, M_{n}=\mathbb{R}^{d}$, $M=E=\mathbb{R}^{d} \times G^{\star}$. Define the mapping $\eta_{\varepsilon}: \mathbb{R}^{d} \rightarrow E$ as $\eta_{\varepsilon}: x \rightarrow\left(x, k_{\varepsilon}(x)\right)$, where $k_{\varepsilon}$ was given by (33). Then $\eta_{\varepsilon}$ is the measurable mapping for every $\varepsilon$. The mapping $\eta_{\varepsilon}$ complements each coordinate $x \in \mathbb{R}^{d}$ with an additional coordinate $k_{\varepsilon}(x)$ from $G^{\star}$ in accordance with (33). Thus, under the mapping $\eta_{\varepsilon}$, the image of all points from $\partial G_{\varepsilon}^{\sharp}$ and from $\left(G_{\varepsilon}^{c}\right)^{\sharp}$ has the same second coordinate equal to $\star \in G^{\star}$. Then $X_{\varepsilon}=\eta_{\varepsilon} \circ Y_{\varepsilon}$ has sample paths in $D_{E}[0, \infty)$. Moreover, $X_{\varepsilon}$ has continuous sample paths in $E$. The semigroup $T(t)$ on $C_{0}(E)$ is the Feller semigroup as was mentioned in the same Remark 2.2. We stress that in this context $C_{0}(M)=C_{0}(E)$.

Thus, all the assumptions of [3, Theorem 2.11] are fulfilled, then these processes convergence in law in the space $D_{E}[0, \infty)$. Theorem 2.2 is completely proved. 


\section{$2.2 \quad L^{2}$-setting}

In this section we consider the limit behaviour of $A_{\varepsilon}$ in $L^{2}$ framework. If we set

$$
\hat{D}\left(A_{\varepsilon}\right)=\left\{f \in H^{1}\left(\mathbb{R}^{d}\right) \cap H^{2}\left(G_{\varepsilon}^{\sharp}\right) \cap H^{2}\left(\mathbb{R}^{d} \backslash G_{\varepsilon}^{\sharp}\right),\left.\varepsilon^{2} \nabla f(x)\right|_{\partial G_{\varepsilon}^{\sharp}} \cdot n^{+}=-\left.\nabla f(x)\right|_{\partial G_{\varepsilon}^{\sharp}} \cdot n^{-}\right\}
$$

where $n^{-}, n^{+}$are respectively the internal and external normal vectors on $\partial G_{\varepsilon}^{\sharp}$, then the operator $A_{\varepsilon}$ with the domain $\hat{D}\left(A_{\varepsilon}\right)$ defined in (36) is a self-adjoint operator in $L^{2}\left(\mathbb{R}^{d}\right)$. The last condition on $f \in \hat{D}\left(A_{\varepsilon}\right)$ in (36) is the condition of continuity of the flux $a_{\varepsilon} \nabla f$ at the boundary $\partial G_{\varepsilon}^{\sharp}$. By the Hille-Yosida theorem, $A_{\varepsilon}$ is a generator of a strongly continuous, positive, contraction semigroup $T_{\varepsilon}(t)$ on $L^{2}\left(\mathbb{R}^{d}\right)$.

We describe now a generator of the limit Markov semigroup. Denote $E=\mathbb{R}^{d} \times G^{\star}$, and equip $G^{\star}$ with the norm $d y+\delta_{\star}(\hat{y})$, where $\delta_{\star}(\hat{y})$ is $\delta$-function at $\star$. Then a function $F \in L^{2}(E)$ can be written in a vector form (see (6))

$$
F(x, \hat{y})=\left(f_{0}(x), f_{1}(x, y)\right), \quad x \in \mathbb{R}^{d}, \hat{y} \in G^{\star}, y \in G
$$

with $f_{0} \in L^{2}\left(\mathbb{R}^{d}\right), f_{1} \in L^{2}\left(\mathbb{R}^{d} \times G\right)$. The norm in $L^{2}(E)$ is given by

$$
\|F\|_{L^{2}(E)}^{2}=\int_{\mathbb{R}^{d}} f_{0}^{2}(x) d x+\int_{\mathbb{R}^{d}} \int_{G} f_{1}^{2}(x, y) d y d x .
$$

Defining an operator $A$ in $L^{2}(E)$ by formulas (7), (8), one can easily check that, with a domain

$$
\hat{D}(A)=\left\{f_{0} \in H^{2}\left(\mathbb{R}^{d}\right), f_{1} \in L^{2}\left(\mathbb{R}^{d} ; H^{2}(G)\right),\left.f_{1}(x, y)\right|_{\partial G}=f_{0}(x)\right\},
$$

the operator $(A, \hat{D}(A))$ is a closed symmetric operator in $L^{2}(E)$. Also, $\hat{D}(A)$ is dense in $L^{2}(E)$.

Lemma 2.3. For any $m>0$ the operator $(m-A, \hat{D}(A))$ is a coercive self-adjoint operator in $L^{2}(E)$.

Proof. Consider the following quadratic form

$$
\Gamma(F)=\int_{\mathbb{R}^{d}}\left|\nabla f_{0}\right|^{2}(x) d x+\int_{\mathbb{R}^{d}} \int_{G}\left|\nabla_{y} f_{1}\right|^{2}(x, y) d y d x+m\|F\|_{L^{2}(E)}^{2}
$$

on $E$ with a domain

$$
D(\Gamma)=\left\{f_{0} \in H^{1}\left(\mathbb{R}^{d}\right), f_{1} \in L^{2}\left(\mathbb{R}^{d} ; H^{1}(G)\right),\left.f_{1}(x, y)\right|_{\partial G}=f_{0}(x)\right\} .
$$

Notice that $\left(f_{1}-f_{0}\right) \in L^{2}\left(\mathbb{R}^{d} ; H_{0}^{1}(G)\right)$ for any $f \in D(\Gamma)$. According to [8, Theorem x.x $]$ there exists a unique self-adjoint operator $\tilde{A}_{m}$ on $E$ that has the following properties: its domain $D\left(\tilde{A}_{m}\right)$ is dense in $L^{2}(E) ; D\left(\tilde{A}_{m}\right)$ belongs to $D(\Gamma) ;\left.\left(\tilde{A}_{m} U, U\right)\right|_{L^{2}(E)}=\Gamma(U, U)$ for any $U \in D\left(\tilde{A}_{m}\right)$. We are going to show that $\tilde{A}_{m}$ coincides with $m-A$.

First we prove that $D\left(\tilde{A}_{m}\right) \subset \hat{D}(A)$. Taking $F \in D\left(\tilde{A}_{m}\right)$ and $U=\left(0, u_{1}(x, y)\right)$ with $u_{1} \in$ $C_{0}^{\infty}\left(\mathbb{R}^{d} ; C_{0}^{\infty}(G)\right)$, and using the relation $\left(\tilde{A}_{m} F, U\right)_{L^{2}(E)}=\Gamma(F, U)$, we obtain $\left(\tilde{A}_{m} F, U\right)_{L^{2}(E)}=$ $\left(\left(m-\Delta_{y}\right) f_{1}, u_{1}\right)$ where the right-hand side is understood as a pairing between $L^{2}\left(\mathbb{R}^{d} ; H^{-1}(G)\right)$ and $L^{2}\left(\mathbb{R}^{d} ; H_{0}^{1}(G)\right)$. This implies that $\left(m-\Delta_{y}\right) f_{1} \in L^{2}\left(\mathbb{R}^{d} \times G\right)$ and thus $f_{1} \in L^{2}\left(\mathbb{R}^{d} ; H^{2}(G)\right)$. Choosing now $U=\left(u_{0}(x), 0\right)$ with $u_{0} \in C_{0}^{\infty}\left(\mathbb{R}^{d}\right)$, we get $m f_{0}-\operatorname{div}\left(\Theta \nabla f_{0}\right) \in L_{\tilde{A}}^{2}\left(\mathbb{R}^{d}\right)$. Therefore, $f_{0} \in H^{2}\left(\mathbb{R}_{\tilde{A}}^{d}\right)$, and $D\left(\tilde{A}_{m}\right) \subset \hat{D}\left(\tilde{A}_{\tilde{A}}\right)$. Moreover, $\tilde{A}_{m} F=(m-A) F$ for any $F \in D\left(\tilde{A}_{m}\right)$.

Since $\tilde{A}_{m}$ is self-adjoint, $D\left(\tilde{A}_{m}\right)=\hat{D}(A)$. This yields the desired statement. 
As a core for the operator $A$ defined by (7) (see [3, Section 1.3] for the definition of a core) we choose the set $D_{A}$ defined in (9). Clearly, $D_{A}$ is a dense subset in $L^{2}(E)$.

The fact that the set $\left\{(m-A) F, F \in D_{A}\right\}$ is dense in $L^{2}(E)$ can be justified in the same was as in the proof of Corollary 1.

Applying again the Hille-Yosida theorem, we conclude that $A$ is a generator of a strongly continuous, positive, contraction semigroup $T(t)$ on $L^{2}(E)$.

The definition of a linear mapping $\pi_{\varepsilon}$ in the $L^{2}$ setting should be slightly modified. Namely, we set

$$
\left(\pi_{\varepsilon}^{H} F\right)(x)= \begin{cases}f_{0}(x), & \text { if } x \in \mathbb{R}^{d} \backslash G_{\varepsilon}^{\sharp} ; \\ \frac{1}{\varepsilon^{d}|G|} \int_{\varepsilon G} f_{1}(\hat{x}+\eta, \xi) d \eta, & \text { if } x \in G_{\varepsilon}^{\sharp},\end{cases}
$$

where $x=\hat{x}+\varepsilon \xi \in G_{\varepsilon}^{\sharp}, \hat{x}=\varepsilon\left[\frac{x}{\varepsilon}\right] \in \varepsilon \mathbb{Z}^{d} \subset \mathbb{R}^{d}, \xi=\left\{\frac{x}{\varepsilon}\right\} \in G$.

Theorem 2.3. For every $F \in L^{2}(E)$

$$
T_{\varepsilon}(t) \pi_{\varepsilon}^{H} F \rightarrow T(t) F \quad\left(\text { i.e. }\left\|T_{\varepsilon}(t) \pi_{\varepsilon}^{H} F-\pi_{\varepsilon}^{H} T(t) F\right\|_{L^{2}\left(\mathbb{R}^{d}\right)} \rightarrow 0\right) \quad \text { for all } t \geq 0
$$

as $\varepsilon \rightarrow 0$.

We use the approximation theorem [Theorem 6.1, Chapter 1, [3]] that was formulated in section 2.1. In order to justify the convergence in (41) it is sufficient to prove the following statement.

Lemma 2.4. Let the generators $A$ and $A_{\varepsilon}$ of the strongly continuous, positive, contraction semigroups $T(t)$ and $T_{\varepsilon}(t)$ be defined by (7) and (1), (2),(36), respectively, and assume that a core $D_{A} \subset L^{2}(E)$ for the generator $A$ is defined by (9), and that a bounded linear transformation $\pi_{\varepsilon}^{H}: L^{2}(E) \rightarrow L^{2}\left(\mathbb{R}^{d}\right)$ is defined by (40).

Then for every $F \in D_{A}$, there exists $F_{\varepsilon} \in D\left(A_{\varepsilon}\right)$ such that

$$
\left\|F_{\varepsilon}-\pi_{\varepsilon}^{H} F\right\|_{L^{2}\left(\mathbb{R}^{d}\right)} \rightarrow 0 \quad \text { and } \quad\left\|A_{\varepsilon} F_{\varepsilon}-\pi_{\varepsilon}^{H} A F\right\|_{L^{2}\left(\mathbb{R}^{d}\right)} \rightarrow 0 \quad \text { as } \varepsilon \rightarrow 0 .
$$

Proof. We first show that for any $F=\left(f_{0}(x), f_{1}(x, y)\right) \in D_{A}$ with a compact support the following limit relations hold:

$$
\left\|\pi_{\varepsilon} F-\pi_{\varepsilon}^{H} F\right\|_{L^{2}\left(\mathbb{R}^{d}\right)} \rightarrow 0, \quad\left\|\pi_{\varepsilon} A F-\pi_{\varepsilon}^{H} A F\right\|_{L^{2}\left(\mathbb{R}^{d}\right)} \rightarrow 0
$$

with $\pi_{\varepsilon}$ define in (14). Indeed, due to the definitions of $\pi_{\varepsilon}$ and $\pi_{\varepsilon}^{H}$, we have $\pi_{\varepsilon} F-\pi_{\varepsilon}^{H} F=0$ on $\mathbb{R}^{d} \backslash G_{\varepsilon}^{\sharp}$. On $G_{\varepsilon}^{\sharp}$ the following estimate holds:

$$
\max _{x \in G_{\varepsilon}^{\sharp}}\left|\left(\pi_{\varepsilon} F\right)(x)-\left(\pi_{\varepsilon}^{H} F\right)(x)\right| \leq \max _{y \in \bar{G}} \max _{\mathcal{R}_{\varepsilon}}\left|f_{1}\left(x^{\prime}, y\right)-f_{1}\left(x^{\prime \prime}, y\right)\right| \underset{\varepsilon \rightarrow 0}{\longrightarrow} 0,
$$

where $\mathcal{R}_{\varepsilon}=\left\{x^{\prime}, x^{\prime \prime} \in \mathbb{R}^{d}:\left|x^{\prime}-x^{\prime \prime}\right| \leq \varepsilon\right\}$; the last convergence is a consequence of of uniform continuity of the function $f_{1}$. This implies the first relation in (43). The second one can be justified in the same way.

We now turn to the proof of Lemma 2.4. As a consequence of formula (16) in Lemma 2.2 we obtain that for any $F=\left(f_{0}, f_{1}\right) \in D_{A}$ there exists $F_{\varepsilon} \in D\left(A_{\varepsilon}\right)$ such that

$$
\left\|F_{\varepsilon}-\pi_{\varepsilon} F\right\|_{L^{2}\left(\mathbb{R}^{d}\right)} \rightarrow 0 \quad \text { and } \quad\left\|A_{\varepsilon} F_{\varepsilon}-\pi_{\varepsilon} A F\right\|_{L^{2}\left(\mathbb{R}^{d}\right)} \rightarrow 0, \quad \text { as } \varepsilon \rightarrow 0 .
$$

here we have also used the fact that any function $F_{\varepsilon}$ with a compact support that belongs to the domain of $A^{\varepsilon}$ in the space $C_{0}\left(\mathbb{R}^{d}\right)$ belongs as well to the domain of $A^{\varepsilon}$ in the space $L^{2}\left(\mathbb{R}^{d}\right)$.

It remains to combine (44) and (43) and to apply the triangle inequality, then the desired statement follows. 
We proceed with the description of the spectrum of the limit operator in (7), and then using the strong convergence of Markov semigroup $T_{\varepsilon}$ in $L^{2}(E)$ obtained above we describe the limit behaviour of the spectrum of operator $A_{\varepsilon}$, as $\varepsilon \rightarrow 0$. The spectrum of the limit problem and the results on convergence of spectra have been previously obtained by V. Zhikov in [9]. Here we partly reprove these results using the semigroup approach presented above.

First we represent $f_{1}(x, \xi)$ in $(9)$ as the sum $f_{1}(x, \xi)=f_{0}(x)+g_{1}(x, \xi)$, then $\left.g_{1}\right|_{\xi \in \partial G}=0$. Then the formula in (8) takes the form:

$$
-A\left(f_{0}(x), f_{1}(x, \xi)\right)=\left(\begin{array}{c}
-\Theta \nabla \nabla f_{0}(x)+\frac{1}{\left|G^{c}\right|} \int_{G} \triangle_{\xi} g_{1}(x, \xi) d \xi \\
-\triangle_{\xi} g_{1}(x, \xi)
\end{array}\right)
$$

The operator $-\triangle_{\xi}$ on $G$ with homogeneous Dirichlet boundary condition has a discrete spectrum $\left\{\beta_{k}\right\}_{k \in \mathbb{N}}, \beta_{k}>0, \beta_{k} \rightarrow \infty$. We denote by $\varkappa_{k}(\xi), k=1,2, \ldots$, the corresponding normalized eigenfunctions and introduce the set $\mathbb{N}^{*}$ of indices $k$ such that $\int_{G} \varkappa_{k}(\xi) d \xi=\left\langle\varkappa_{k}\right\rangle \neq 0$.

Lemma 2.5. The continuous spectrum $\sigma_{\text {cont }}(-A)$ of the operator $-A$ is a countable set of nonoverlapping segments

$$
\sigma_{\text {cont }}(-A)=\bigcup_{k \in \mathbb{N}^{*}}\left[\tilde{\lambda}_{k}, \beta_{k}\right]
$$

where $\tilde{\lambda}_{1}=0$, and $\tilde{\lambda}_{k}, k>1$, is the nearest to $\beta_{k}$ solution of equation

$$
\frac{1}{\left|G^{c}\right|} \sum_{k} \frac{\alpha_{k}^{2} \beta_{k}}{\beta_{k}-\tilde{\lambda}}+1=0 \quad \text { with } \quad \alpha_{k}=<\varkappa_{k}>
$$

such that $\tilde{\lambda}_{k}<\beta_{k}$. The discrete spectrum of the operator $-A$ is the union of eigenvalues $\beta_{k}$ with $k \in \mathbb{N} \backslash \mathbb{N}^{*}$.

Proof. The second line in the equation $-A F=\lambda F$ reads

$$
-A\left(f_{0}(x)+g_{1}(x, \xi)\right)=-\triangle_{\xi} g_{1}(x, \xi)=\lambda\left(f_{0}(x)+g_{1}(x, \xi)\right) .
$$

The function $f_{0}(x)$ does not depend on $\xi$, its Fourier series w.r.t. $\varkappa_{k}(\xi)$ takes the form $f_{0}(x) \cdot 1=$ $f_{0}(x) \sum_{k} \alpha_{k} \varkappa_{k}(\xi)$. Denoting by $\gamma_{k}$ the Fourier coefficients of $g_{1}$, from (46) we get

$$
-\triangle_{\xi} g_{1}(x, \xi)=\sum_{k} \beta_{k} \gamma_{k} \varkappa_{k}(\xi)=\lambda f_{0}(x) \sum_{k} \alpha_{k} \varkappa_{k}(\xi)+\lambda \sum_{k} \gamma_{k} \varkappa_{k}(\xi) .
$$

Consequently, for any $\lambda \notin\left\{\beta_{k}\right\}$ we have $\gamma_{k}=\lambda f_{0}(x) \frac{\alpha_{k}}{\beta_{k}-\lambda}$, and thus the function

$$
g_{1}(\xi)=\sum_{k} \gamma_{k} \varkappa_{k}(\xi)=\lambda f_{0}(x) \sum_{k} \frac{\alpha_{k}}{\beta_{k}-\lambda} \varkappa_{k}(\xi),
$$

is a solution of equation $-A\left(f_{0}+g_{1}\right)=-\triangle_{\xi} g_{1}=\lambda\left(f_{0}+g_{1}\right)$ for any $\lambda \notin\left\{\beta_{k}\right\}$.

Inserting (47) in the first line of the equation $-A F=\lambda F$ yields

$$
-\Theta \nabla \nabla f_{0}(x)-\lambda f_{0}(x) \frac{1}{\left|G^{c}\right|} \sum_{k} \frac{\alpha_{k} \beta_{k}}{\beta_{k}-\lambda} \int_{G} \varkappa_{k}(\xi) d \xi=\lambda f_{0}(x) .
$$


We conclude that

$$
-\Theta \nabla \nabla f_{0}(x)=\lambda f_{0}(x)\left(\frac{1}{\left|G^{c}\right|} \sum_{k} \frac{\alpha_{k} \beta_{k}}{\beta_{k}-\lambda}<\varkappa_{k}>+1\right)=\lambda f_{0}(x)\left(\frac{1}{\left|G^{c}\right|} \sum_{k} \frac{\alpha_{k}^{2} \beta_{k}}{\beta_{k}-\lambda}+1\right) .
$$

Since the spectrum of the operator $-\Theta \nabla \nabla$ fills up the positive half-line, we obtain that all $\lambda>0$ such that

$$
\frac{1}{\left|G^{c}\right|} \sum_{k} \frac{\alpha_{k}^{2} \beta_{k}}{\beta_{k}-\lambda}+1 \geq 0
$$

belong to the spectrum of the operator $-A$. One can easily check that the segment $\left[0, \beta_{1}\right]$ belongs to the continuous spectrum of $-A$. This implies the desired statement on $\sigma_{\text {cont }}(-A)$.

It is straightforward to check that for all $k \in \mathbb{N} \backslash \mathbb{N}^{*}$ the functions $\left(0, \varkappa_{k}(\xi)\right)$ are eigenfunctions of $-A$. This completes the proof.

Proposition 2.1. If $\lambda \in \sigma(-A)$, then $\lambda$ is the limit of a sequence of points from the spectra of operators $A_{\varepsilon}$, i.e. $\forall \delta>0 \exists \varepsilon_{0}>0$ such that there exists $\lambda_{\varepsilon} \in \sigma\left(-A_{\varepsilon}\right) \cap O_{\delta}(\lambda)$ for all $\varepsilon<\varepsilon_{0}$.

Proof. Since $\lambda \in \sigma(-A)$, there exist functions $F_{n} \in D_{A}$ with $\left\|F_{n}\right\|=1$ such that $\left\|(A-\lambda) F_{n}\right\| \rightarrow 0$ as $n \rightarrow \infty$. Using the approximation theorem we additionally have that for any $F_{n} \in D_{A}$ there exists $F_{n}^{\varepsilon} \in D\left(A_{\varepsilon}\right)$ such that

$$
\left\|F_{n}^{\varepsilon}-F_{n}\right\| \rightarrow 0 \quad \text { and } \quad\left\|A_{\varepsilon} F_{n}^{\varepsilon}-A F_{n}\right\| \rightarrow 0 \quad \text { as } \varepsilon \rightarrow 0 .
$$

Thus we obtain that for any (small) $\delta>0$ there exists $\varepsilon_{0}=\varepsilon_{0}(\lambda)>0$ such that

$$
\left\|A_{\varepsilon} F_{n}^{\varepsilon}-\lambda F_{n}^{\varepsilon}\right\|<\delta \quad \text { for all } \quad \varepsilon<\varepsilon_{0} .
$$

This implies that there is a point of the spectrum of $A_{\varepsilon}$ in the $\delta$-neighbourhood of $\lambda$.

\section{References}

[1] G. Allaire, Homogenization and two-scale convergence. SIAM J. Math. Anal. 23(6) (1992), 14821518.

[2] T. Arbogast; J.J. Douglas; U. Hornung, Derivation of the double porosity model of single phase flow via homogenization theory. SIAM J. Math. Anal., 21(4) (1990), 823-836.

[3] S. N. Ethier, T. G. Kurtz, Markov processes: Characterization and convergence. Wiley \& Sons, 2005.

[4] M. Fukushima; Y. Ōshima; M. Takeda, Dirichlet forms and symmetric Markov processes., De Gruyter Studies in Mathematics, 19. Walter de Gruyter \& Co., Berlin, 1994.

[5] V.V. Jikov; S.M. Kozlov; O.A. Oleinik, Homogenization of differential operators and integral functionals., Springer-Verlag, Berlin, 1994.

[6] V.A. Marchenko; E.Ya. Khruslov, Homogenization of partial differential equations. Progress in Mathematical Physics, 46. Birkhuser Boston, 2006.

[7] A. Piatnitski; E. Zhizhina, Scaling limit of symmetric random walk in high-contrast periodic environment. J. Stat. Phys., 169(3) (2017), 595-613. 
[8] M. Reed; B. Simon, Methods of modern mathematical physics. IV. Analysis of operators., Academic Press, New York-London, 1978.

[9] V.V. Zhikov, On an extension and an application of the two-scale convergence method. Sb. Math., 191(7-8) (2000), 973-1014.

[10] V.V. Zhikov, Weighted sobolev spaces. Sb. Math., 189(8) (1998), 1139-1170.

[11] V.V. Zhikov, Two-scale convergence ans spectral questions of the homogenization theory. $J$. Math. Sci., 114(4) (2003), 1450-1460. 